\title{
Searches for new physics in events with top-quark pairs in ATLAS
}

\author{
Olaf Nackenhorst ${ }^{* \dagger}$ \\ TU Dortmund University \\ E-mail: olaf.nackenhorstecern.ch
}

\begin{abstract}
Many theories beyond the Standard Model (SM) predict new heavy particles that would preferably couple to top quarks. In several direct searches it is assumed that these particles decay to final states with a pair of top quarks. This results either in various signatures with top-quark pairs or in a resonant distribution of the invariant mass of the top-quark pair. In indirect searches it is assumed that SM processes might be affected by massive new particles, such as the enhancement of the rare SM process of four-top-quark production. The results from latest searches, performed by the ATLAS Collaboration with $13 \mathrm{TeV}$ data, for vector-like-quark pair-production, for resonances that decay to a top-quark pair and for four-top-quark production are presented.
\end{abstract}

7th Annual Conference on Large Hadron Collider Physics - LHCP2019

20-25 May, 2019

Puebla, Mexico

\footnotetext{
* Speaker.

${ }^{\dagger}$ on behalf of the ATLAS collaboration
} 


\section{Introduction}

The first two runs of the LHC physics program have been a tremendous success for the collaborations of the two general purpose detectors ATLAS [1] and CMS [2] and their results have been so far well in agreement with the predictions of the Standard Model of particle physics (SM). However, there are still many open questions, which cannot be answered by the SM, such as "What is the origin of dark matter?", "Why is the mass of the Higgs boson so much smaller than the Planck scale?" or "How can gravity be included in the SM?". Hence, we know there must be physics beyond the SM (BSM). Since the top quark is the heaviest particle in the SM, many BSM theories predict new heavy particles with a strong coupling to top quarks. The decay of these new particles would consequently often result in final states with top quarks. Furthermore, top-quark pairs are produced copiously at the LHC, which allows for precise measurements of the properties of the top quark. Any deviations in these measurements from the predictions of the SM would give us a hint of new physics. As a consequence, there is a rich search program at ATLAS for new physics in events with top-quark pairs. In direct searches it is assumed that a new heavy particle is produced, which decays to signatures with top quarks. Several searches for vector-like-quark pair production will be discussed as examples of such signatures. If the heavy particle decays directly into a pair of top quarks, a search for bumps in the invariant mass spectrum of the $t \bar{t}$-system can be performed, which will also be presented in the following. In indirect searches the hypothetical new heavy particle affects well-known SM processes through loop corrections, which can for instance enhance the production of rare processes such as the four-top-quark production. The current searches for four-top-quark production will also be briefly summarized.

\section{Searches for vector-like-quark pair-production}

Vector-like quarks (VLQs) are often introduced as a solution to the hierarchy problem of the Higgs-boson mass because they cancel the quadratic divergences of the loop corrections of the SM particles. They are predicted in extensions to the SM like Little Higgs [3, 4], Composite Higgs [5, 6] or warped extra dimensions [7]. Their left- and right-handed chiral representations transform the same way under the $\mathrm{SU}(2)$ symmetry and hence their masses are not caused by the coupling to the Higgs boson. Since in many models the mixing with SM quarks is proportional to the quark mass, VLQs couple predominantly to the bottom and top quark of the SM. As a consequence, searches often consider only the vector-like quark partner of the bottom and top quark with SM charge $(B$ and $T$ ) or with an exotic charge of $+5 / 3$ and $-4 / 3(X$ and $Y$ ). VLQs are predicted to be produced either in pairs via the strong interaction or singly through the weak interaction. Since they can also decay via flavour-changing neutral-currents, the vector-like $T$ is allowed to decay either into $W b$, $Z t$ or $H t$, while the vector-like $B$ can decay into $W t, Z b$ or $H b$. The branching ratios depend on the VLQ mass and the realization as an electroweak singlet, doublet or triplet.

Due to the various decay possibilities, the resulting final state of the VLQ pair decay is manifold and the ATLAS collaboration addresses all signatures in dedicated searches [8-14]. In the following, only the three latest searches for the $Z(\ell \ell) t / b+X$ [12], the trilepton / same-sign dilepton [11] and the fully hadronic [13] final states will be presented, which all address both the $B \bar{B}$ and $T \bar{T}$ decay. 


\subsection{Search for $Z(\ell \ell) t / b+X$ final state}

The $Z(\ell \ell) t / b+X$ analysis [11] focuses on VLQ pair decays with two opposite-sign leptons and at least two $b$-tagged jets or at least three leptons and at least one $b$-tagged jet. In both lepton channels a $Z$-boson candidate reconstructed from the leptons is required to have a large transverse momentum. In the two-lepton channel, events are further categorized depending on the number of large- $R$ jets $(0 \mathrm{~J}, 1 \mathrm{~J}, \geq 2 \mathrm{~J})$ in three signal regions. For the $\leq 1 \mathrm{~J}$ and $\geq 2 \mathrm{~J}$ region, two control regions are defined for the most important background processes, $t \bar{t}$ and $Z+$ jets. Similarly, in the three-lepton channel two control regions are defined for the dominant diboson $(W W / Z Z / W Z)$ and $t \bar{t}+X(X=W / Z / W W / t \bar{t})$ background. The definitions of the control regions in both channels impose various cuts on the scalar sum of the transverse momenta of all jets, $H_{\mathrm{T}}$, the transverse momentum, $p_{T, \ell \ell}$, and mass, $m_{\ell \ell}$, of the $Z$-boson candidate and missing transverse energy, $E_{\mathrm{T}}^{\mathrm{miss}}$. A profile likelihood fit in all signal and control regions is performed using as disciminant $H_{\mathrm{T}}$ in the $0 \mathrm{~J}$ and $1 \mathrm{~J}$ region, the invariant mass of the $Z$-boson candidate and the highest- $p_{\mathrm{T}} b$-tagged jet, $m_{Z b}$, in the $\geq 2 \mathrm{~J}$ region (see Figure 1 (a)) and the scalar sum of the jet and lepton transverse momenta, $S_{T}$, in the three-lepton channel. In the absence of a significant excess of data events above the SM predictions, 95\% confidence level (CL) lower limits on the $T$ and $B$ mass are set depending on the branching ratios of the possible decays, which is shown for the $T \bar{T}$ decay in Figure 1 (b). Assuming exclusive decays of $T \rightarrow Z t$ and $B \rightarrow Z b$, for which the search is most sensitive, lower limits of $m_{T}>1340 \mathrm{GeV}$ and $m_{B}>1220 \mathrm{GeV}$ are observed, respectively.

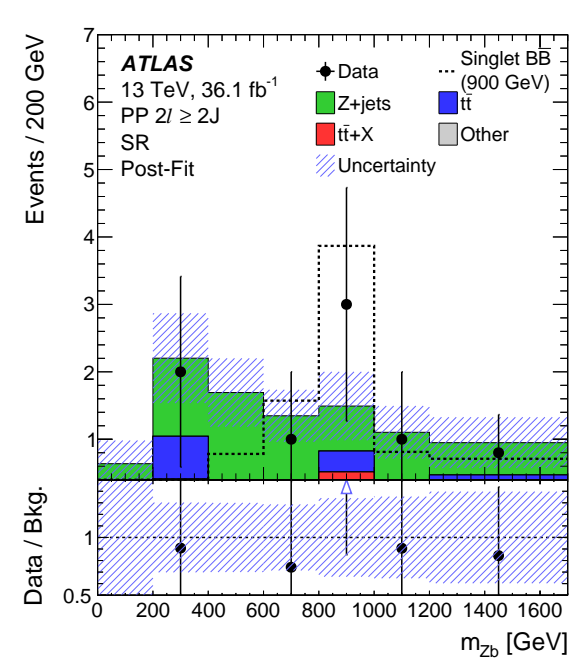

(a)

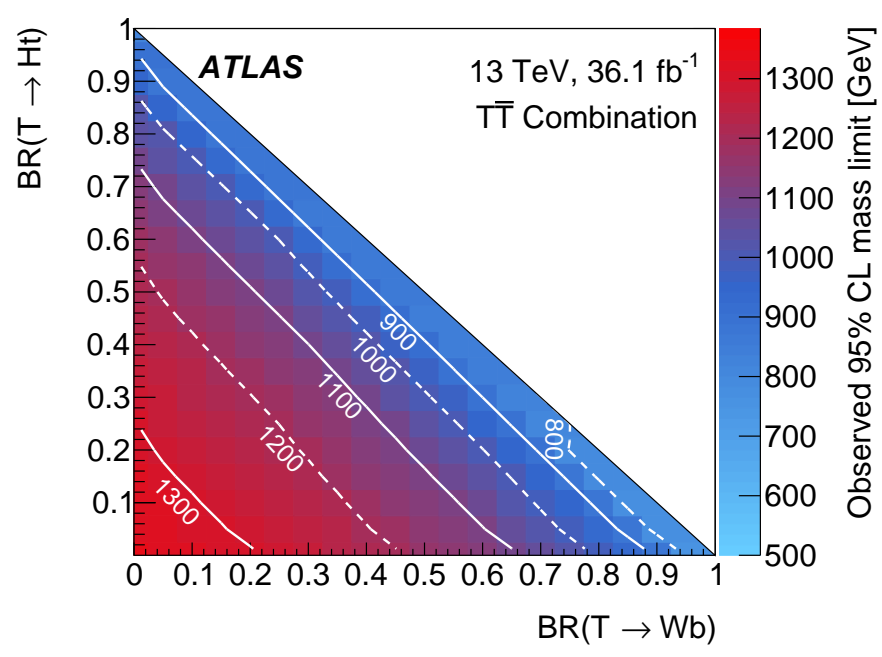

(b)

Figure 1: (a) The post-fit distribution of the invariant mass of the $\mathrm{Z}$ boson candidate and the highest- $p_{\mathrm{T}}$ $b$-tagged jet, $m_{Z b}$, between data and the background prediction in the most sensitive signal region $(\geq 2 \mathrm{~J})$ of the two lepton channel. (b) Observed 95\% CL lower limits from the combination of the pair-production channels on the mass of vector-like quarks for all combinations of branching ratios (BR) [11].

\subsection{Search for the trilepton / same-sign dilepton final state}

The trilepton / same-sign dilepton analysis [11] addresses VLQ pair decays with either two 
same-sign leptons or at least three leptons in the final state with additional $b$-tagged jets. Events are divided into eight signal and six validation regions depending on the number of jets, $b$-tagged jets, $H_{\mathrm{T}}$ and $E_{\mathrm{T}}^{\text {miss }}$ criteria. The most important background is estimated using a data-driven approach and is originating from fake/non-prompt leptons and leptons with misidentified charge. Following a cut-and-count approach the expected event yields in each signal region are simultaneously fit to the observed data events and the improved predictions are verified in the validation regions. Since no significant excess above the SM prediction is observed in data, lower limits on the VLQ masses are set as a function of the branching ratios. The search is most sensitive in the $T \rightarrow W b$ and $B \rightarrow W t$ decays and observed limits of $m_{T}>980 \mathrm{GeV}$ and $m_{B}>1000 \mathrm{GeV}$ are set for the singlet model.

\subsection{Search for the fully hadronic final state}

The fully-hadronic analysis [13] targets all VLQ pair decays with fully hadronic final states by requiring at least four high- $p_{\mathrm{T}}$ jets, of which at least two are $b$-tagged, at least two large- $R$ reclusted jets with variable radius and high $H_{\mathrm{T}}$. The overwhelming multi-jet background, which is estimated using a data-driven approach, is suppressed by making use of a multi-class boosted object tagger. This tagger uses a deep neural network to unambiguously identify the origin of the large- $R$ jet to be either a $V$-boson ( $W / Z$-boson), a Higgs boson, a top quark or a background object. The discriminant function of the tagger is shown in Figure 2 (a) as an example for the Higgs boson identification. Events are categorized into twelve different signal regions based on the number

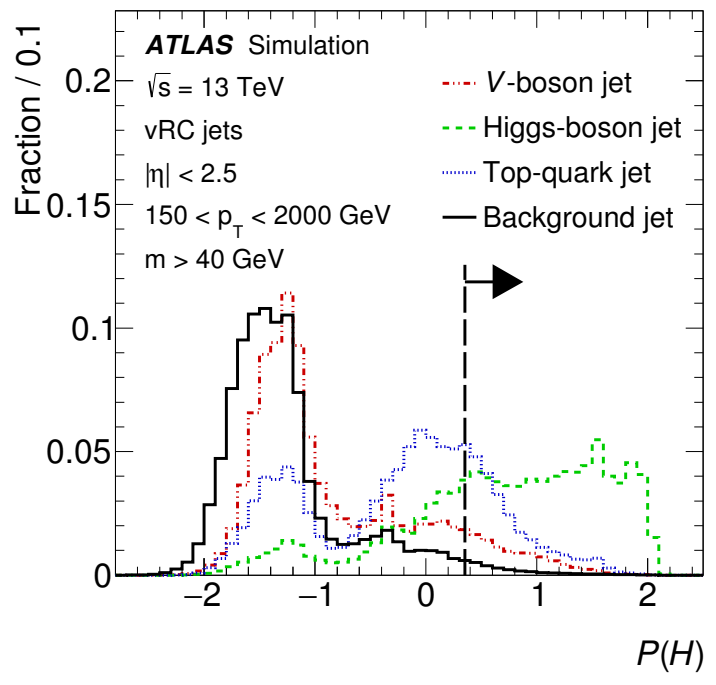

(a)

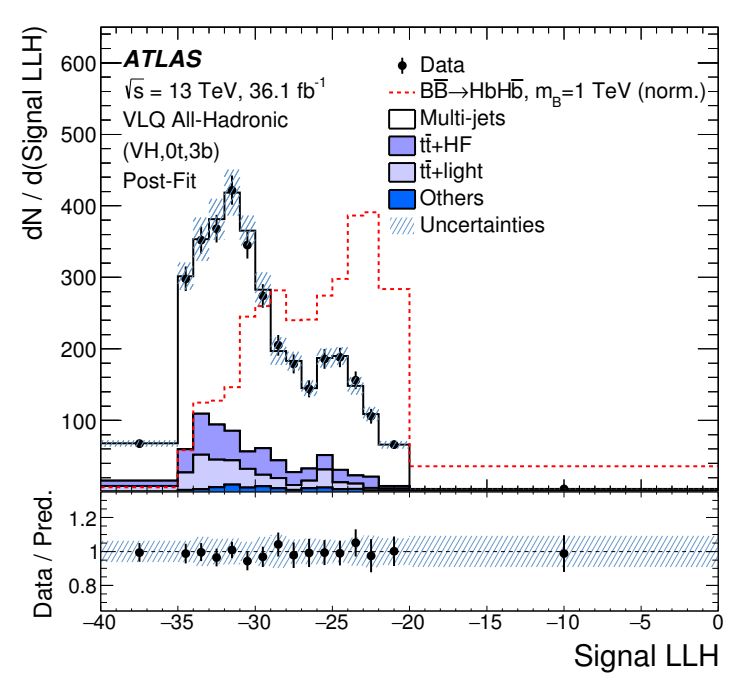

(b)

Figure 2: (a) The discriminant function $P$ for the Higgs-tagger. Signal jets ( $V$-boson, Higgs boson, top-quark jets) are defined by matching the large- $R$ jet to the corresponding object at generator level. (b) Comparison between data and prediction for the signal LLH distribution after the fit to the data under the backgroundonly hypothesis for one of the most sensitive signal regions $(\mathrm{VH}, 0 \mathrm{t}, 3 \mathrm{~b})$. The contribution labeled 'Others' is the combination of single-top-quark and $t \bar{t}+X$ backgrounds [13]. 
of $V$-boson, Higgs boson and top-quark tags as well as the number of $b$-tagged jets. In order to further distinguish VLQ pair events from multi-jet events, the matrix element method is employed in order to calculate in each signal region a signal log-likelihood for the possible VLQ pair decays. Using this variable (see Figure 2 (b)) as a discriminant in a profile likelihood fit, lower limits on the VLQ masses are set, depending on the branching ratio. The highest sensitivity is obtained for $T \rightarrow H t$ and $B \rightarrow H b$ decays, for which for both VLQ types a limit of $m_{T}>1010 \mathrm{GeV}$ is observed.

\subsection{Combination of searches for VLQ pair production}

The ATLAS collaboration has also performed a combination [15] of all searches for pairproduced VLQs [8-14] in order to improve the sensitivity. The results of the combined profile likelihood fit is limited by statistical uncertainties. Observed (expected) lower limits on the VLQ masses of $m_{T}>1310$ (1220) GeV and $m_{B}>1030$ (980) GeV are obtained for an arbitrary combination of branching ratio, which nicely demonstrates the complementarity of the various searches contributing in different branching-ratio corners. Nevertheless, the combination is most sensitive to the $T \rightarrow H t$ and $B \rightarrow W t$ decays, for which even higher mass limits are obtained, as shown in Figure 3.

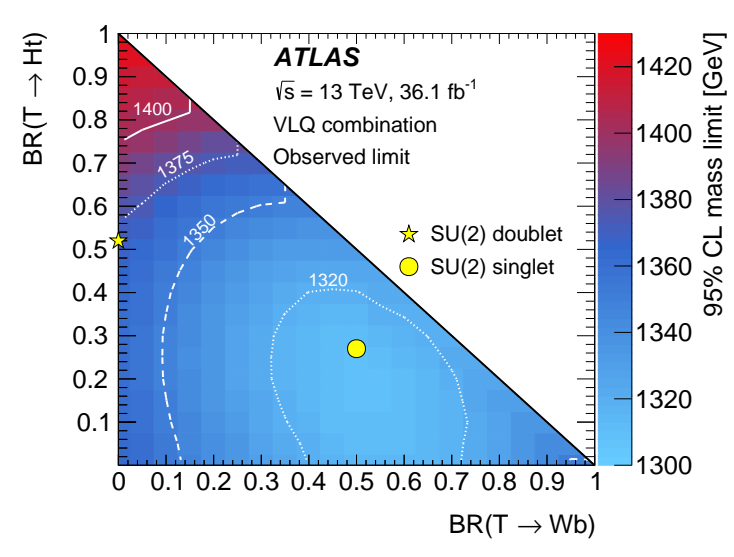

(a)

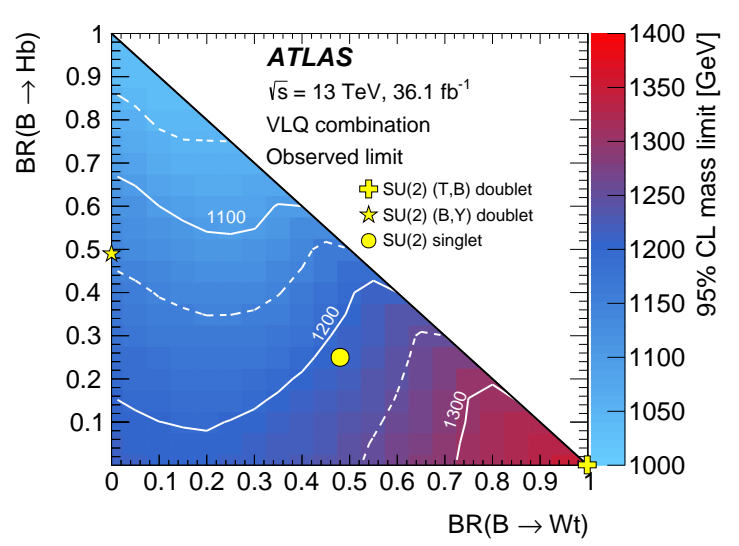

(b)

Figure 3: Observed lower limits at $95 \% \mathrm{CL}$ on the mass of the (a) $\mathrm{T}$ and (b) B as a function of the branching ratios. The yellow markers indicate the branching ratios for the $\mathrm{SU}(2)$ singlet and doublet scenarios for VLQ masses above $800 \mathrm{GeV}$ where the branching ratios become approximately independent of the VLQ mass [15].

\section{Searches for $t \bar{t}$ resonances}

Several BSM models predict new heavy particles that can directly decay into a pair of top quarks. If these new particles exist, they would result in a resonance in the continuously falling mass spectrum of the $t \bar{t}$-system. When searching for such bumps it is hence essential to reconstruct the decay of the top-quark pair. The reconstruction is performed in a resolved channel, where it is assumed that all decay products of the top quarks are isolated, or in a boosted channel, where 
several decay products might overlap and are reconstructed as a single object. Several hypothetical particles predicted by benchmark models are tested in these searches. The first is a spin- 1 coloursinglet, usually referred to as $Z^{\prime}$-boson, which is predicted by topcolor-assisted technicolor (TC2) models [16, 17], and by simplified models of dark matter (DM) [18]. In the TC2 models the $Z^{\prime}$ boson is leptophobic and couples only to first- and third-generation quarks, while in the dark matter models the $Z^{\prime}$-boson can be an axial-vector or vector mediator [19]. The second benchmark particle is a spin-1 colour-octet, which can be a Kaluza-Klein excitation of the gluon, $g_{K K}$, as described in Randall-Sundrum models with a single warped extra dimension [20, 21]. The last tested particle is a spin-2 colour-singlet, such as the Kaluza-Klein excitation of the graviton, $G_{K K}$, as postulated in a bulk Randal-Sundrum model with an extra dimension [22-24]. The ATLAS collaboration has performed searches for these resonances in the zero-lepton [25] and one-lepton [26] channel of the $t \bar{t}$ decay.

\subsection{One-lepton channel}

In the boosted region of the one-lepton channel [26] a leptonically-decaying top-quark candidate is reconstructed by finding a high- $p_{\mathrm{T}}$ jet close to the lepton within $\Delta R<1.5$ and combining this jet with the lepton and $E_{\mathrm{T}}^{\text {miss }}$ information using a $W$-boson mass constraint. The hadronicallydecaying top quark is simply obtained by identifying a top-tagged large- $R$ jet using its mass and $N$-subjettiness [27], which is well enough separated from the leptonic top-quark candidate. The invariant mass of the $t \bar{t}$-system $m_{t \bar{t}}$ is then simply obtained by combining the leptonic and hadronic top-quark candidate. In the resolved region, which is orthogonal to the boosted region, at least four jets with $p_{\mathrm{T}}>25 \mathrm{GeV}$ are required, from which $m_{t \bar{t}}$ is reconstructed using a $\chi^{2}$-method with a top-quark and $W$-boson mass constraint and transverse momentum balance is imposed on the leptonically- and hadronically-decaying top quark. Each of the boosted and resolved regions is divided into six signal regions based on the lepton flavour $(\mathrm{e} / \mu)$ and whether the leptonic and/or hadronic top-quark candidate can be matched to a $b$-tagged track jet. The $m_{t \bar{t}}$ distribution in each region is then used to test for the benchmark particles. No significant excess above the SM prediction is observed in data, and lower limits on the masses of the hypothetical particles are set, which are summarized in Table 1.

Table 1: Summary of the excluded mass ranges at 95\% CL for the various benchmark models [26].

\begin{tabular}{llcc}
\hline Signal & & Expected excluded mass $[\mathrm{TeV}]$ & Observed excluded mass $[\mathrm{TeV}]$ \\
\hline$Z_{\mathrm{TC} 2}^{\prime}$ & $(\Gamma=1 \%)$ & $<3.0$ & $<2.6$ \\
\hline \multirow{2}{*}{$Z_{\text {med }}^{\prime}$} & $($ vector $)$ & $<1.4$ & $<1.6$ \\
& $($ axial-vector $)$ & $<1.2$ & $<1.4$ \\
\hline \multirow{2}{*}{$g_{\mathrm{KK}}$} & $(\Gamma=15 \%)$ & $<3.8$ & $<3.5$ \\
& $(\Gamma=30 \%)$ & $<3.7$ & $<3.2$ \\
\hline \multirow{2}{*}{$G_{\mathrm{KK}}$} & & {$[0.45,0.65]$} & {$[0.45,0.65]$} \\
\hline
\end{tabular}




\subsection{Zero-lepton channel}

In the zero-lepton channel [25] the boosted region is defined by requiring at least two high- $p_{\mathrm{T}}$ large- $R$ jets, which are matched to $b$-tagged track jets. The invariant mass $m_{t \bar{t}}$ is reconstructed by top-tagging the large- $R$ jets based on their mass and n-subjettiness ratio $\tau_{32}$ [27]. The events are then classified into eight signal regions based on the number of $b$-tagged track jets and the value of a $\tau_{32}$-likelihood ratio of signal and background events. The resolved region is defined by requiring at least six high- $p_{\mathrm{T}}$ jets, of which at least two are $b$-tagged. The bucket of tops method [28] using top-quark and $W$-boson mass constraints is used in order to reconstruct the $t \bar{t}$-system. Based on these buckets one signal and three control regions are defined. The multi-jet background, which is the only relevant background apart from $t \bar{t}$ events, is estimated using a data-driven approach. Since no significant bump of data events is seen in the $m_{t \bar{t}}$ distributions of all regions, mass ranges are excluded for the hypothetical particles of the benchmark models, as summarized in Table 2.

Table 2: Summary of the excluded mass ranges at 95\% CL for the various benchmark models [25].

\begin{tabular}{clcc}
\hline Signal & & Expected excluded mass $[\mathrm{TeV}]$ & Observed excluded mass $[\mathrm{TeV}]$ \\
\hline \multirow{2}{*}{$Z_{\mathrm{TC} 2}^{\prime}$} & $(\Gamma=1 \%)$ & {$[0.57,2.8]$} & {$[0.58,3.1]$} \\
& $(\Gamma=3 \%)$ & {$[0.51,3.6]$} & {$[0.53,3.6]$} \\
\hline \multirow{2}{*}{$Z_{\text {med }}^{\prime}$} & $($ vector $)$ & {$[0.75,1.07] \cup[2.0,2.1]$} & {$[0.74,0.97] \cup[2.0,2.2]$} \\
& $($ axial-vector $)$ & {$[1.99,2.04]$} & {$[0.80,0.92] \cup[2.0,2.2]$} \\
\hline \multirow{2}{*}{$g_{\mathrm{KK}}$} & $(\Gamma=10 \%)$ & $<3.5$ & $<3.4$ \\
& $(\Gamma=20 \%)$ & $<3.4$ & $<3.4$ \\
& $(\Gamma=30 \%)$ & $<3.3$ & $<3.4$ \\
& $(\Gamma=40 \%)$ & $<3.2$ & $<3.4$ \\
\hline
\end{tabular}

\section{Searches for four-top-quark production}

Four-top-quark production is a rare SM process, for which the NLO production cross section in $p p$ collisions at $\sqrt{s}=13 \mathrm{TeV}$ is predicted to be $9.2 \mathrm{fb}$ [29]. However, the production rate could be enhanced by several BSM processes. In the context of the searches performed by the ATLAS collaboration [11], three different BSM benchmark models are tested for such an enhancement. The first is an effective field theory (EFT) approach, in which a four-point contact interaction (CI) is introduced. The second is based on a model with two compactified universal extra-dimension (2UED) [30], in which the four top quarks are produced through a chain decay of various KaluzaKlein excitations, which also results in many additional leptons and jets. The last production model is a two Higgs doublet model (2HDM) [31], in which another heavy scalar or pseudo-scalar Higgs is produced in association with a top-quark pair, which subsequently decays into a second pair of top quarks. The ATLAS collaboration has searched for four-top-quark production in the trilepton / same-sign dilepton channels [11] and in the one or two opposite-sign lepton channels [32]. 


\subsection{Trilepton / same-sign dilepton channel}

The search in the trilepton / same-sign dilepton channel [11] is the same analysis as earlier introduced in the context of the VLQ searches but is now reinterpreted for SM and BSM four-topquark production. The search is not sensitive to the SM production yet, but an upper limit of 69.2 $\mathrm{fb}$ is observed on the cross section at $95 \% \mathrm{CL}$, while a limit of $29.0_{-8.1}^{+12.2} \mathrm{fb}$ was expected from simulations. For the CI model, the observed (expected) limit is 38.6 (20.8) fb, which can also be interpreted as a limit on the interaction strength $C_{4 t}$ as a function of the cut-off scale $\Lambda$, as shown in Figure 4 (a). In the symmetric case of the 2UED model, a limit on the Kaluza-Klein mass of $m_{K K}>1.45 \mathrm{TeV}$ is set, and for the 2HDM limits on the heavy (pseudo-)scalar Higgs mass are set for various values of $\tan \beta$ as presented in Figure 4 (b).

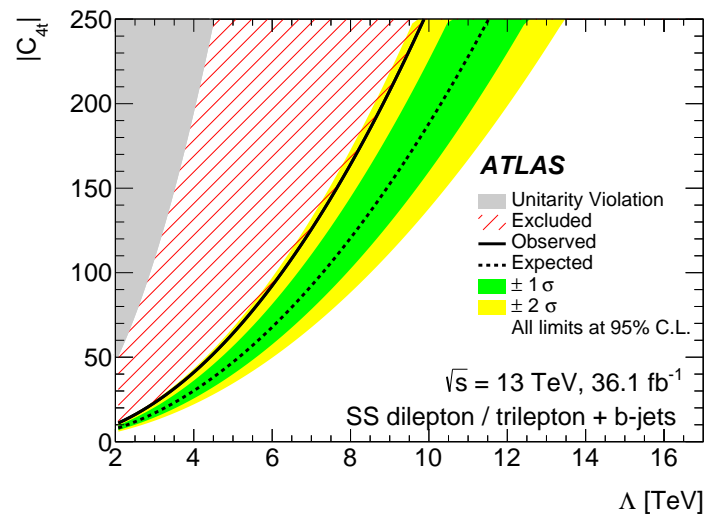

(a)

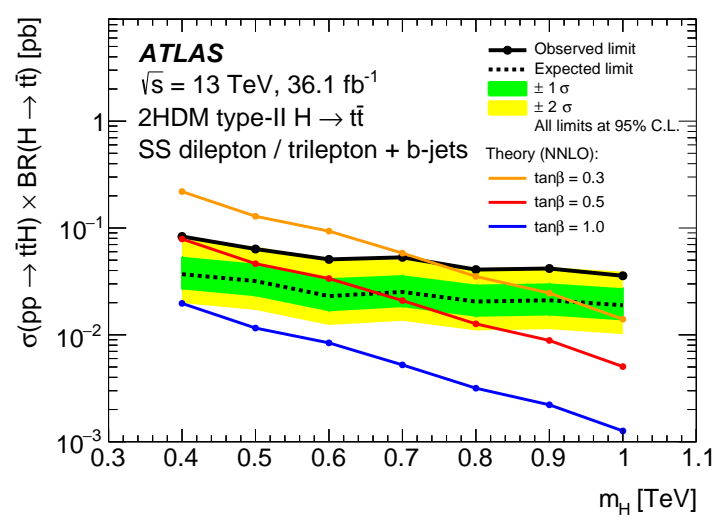

(b)

Figure 4: (a) Constraints in the $\left(\left|C_{4 t}\right|, \Lambda\right)$ plane. (b) Limits on the cross-section of the four-top-quark production through a heavy scalar Higgs boson times the branching ratio for the Higgs boson to decay into $t \bar{t}$. Theoretical predictions for three values of $\tan \beta$ are shown [11].

\subsection{One or two opposite-sign lepton channel}

The other search [32] targets final states with exactly one lepton or two opposite-sign leptons in addition to many jets, of which several are $b$-tagged. Events are categorized into 20 signal and 16 validation regions based on the number of jets, $b$-tagged jets and mass-tagged reclustered large- $R$ jets. Since the signal regions are required to have high jet and $b$-tagged jet multiplicities, the $t \bar{t}+$ jets background is estimated from data using the tag-rate-function method [33]. Using this method, the $b$-tagging efficiencies of a jet are measured in regions with lower jet multiplicities and then applied to lower $b$-tagging multiplicities in order to extrapolate the shape and yield of $t \bar{t}$ events to the very high multiplicities of this analysis. As a final discriminant $H_{\mathrm{T}}$ is then used in a profile likelihood fit of all signal regions in order to extract a signal strength of the SM expectation of the four-top-quark production. As the search is not sensitive to the SM production, an observed (expected) upper limit on the SM production cross section of 47 (33) fb is set. In the combination with the trilepton / same-sign dilepton channel the observed (expected) limit via a CI is 21 (15) fb. 


\section{Summary and Conclusion}

The ATLAS collaboration has a broad search program for new physics with top-quark pairs; however, there is no sign for new physics yet. In the searches for VLQ pair production all branching ratios to SM particles are covered and VLQ masses above the TeV scale can be excluded for any branching ratios. Both of the lepton channels of the $t \bar{t}$ resonance search have comparable sensitivity and can exclude several exotic benchmark particles up to masses of roughly $4 \mathrm{TeV}$. The search for four-top-production focuses currently on final states with leptons and has not seen an enhancement of the SM production caused by new heavy particles. However, most of the presented searches are not yet using the full Run 2 data set, and hence the full potential of the recorded data is not yet exploited.

\section{References}

[1] ATLAS Collaboration, The ATLAS Experiment at the CERN Large Hadron Collider, JINST 3 (2008) S08003.

[2] CMS Collaboration, The CMS experiment at the CERN LHC, JINST 3 (2008) S08004.

[3] N. Arkani-Hamed, A. G. Cohen, E. Katz, and A. E. Nelson, The Littlest Higgs, JHEP 07 (2002) 034, arXiv: hep-ph/0206021 [hep-ph] .

[4] M. Schmaltz and D. Tucker-Smith, Little Higgs Theories, Ann. Rev. Nucl. Part. Sci. 55 (2005) 229, arXiv: hep-ph/0502182 [hep-ph] .

[5] D. B. Kaplan, H. Georgi, and S. Dimopoulos, Composite Higgs scalars, Phys. Lett. B 136 (1984) 187.

[6] K. Agashe, R. Contino, and A. Pomarol, The minimal composite Higgs model, Nucl. Phys. B 719 (2005) 165, arXiv: hep-ph/ 0412089 [hep-ph] .

[7] L. Randall and R. Sundrum, Large Mass Hierarchy from a Small Extra Dimension, Phys. Rev. Lett. 83 (1999) 3370, arXiv: hep-ph/9905221 [hep-ph] .

[8] ATLAS Collaboration, Search for pair production of up-type vector-like quarks and for four-top-quark events in final states with multiple b-jets with the ATLAS detector, JHEP 07 (2018) 089, arXiv: 1803.09678 [hep-ex] .

[9] ATLAS Collaboration, Search for pair production of heavy vector-like quarks decaying to high- $p_{T} W$ bosons and $b$ quarks in the lepton-plus-jets final state in pp collisions at $\sqrt{s}=$ $13 \mathrm{TeV}$ with the ATLAS detector, JHEP 10 (2017) 141, arXiv: 1707.03347 [hep-ex ] .

[10] ATLAS Collaboration, Search for pair production of vector-like top quarks in events with one lepton, jets, and missing transverse momentum in $\sqrt{s}=13 \mathrm{TeV}$ pp collisions with the ATLAS detector, JHEP 08 (2017) 052, arXiv: 1705.10751 [hep-ex ] .

[11] ATLAS Collaboration, Search for new phenomena in events with same-charge leptons and $b$-jets in pp collisions at $\sqrt{s}=13 \mathrm{TeV}$ with the ATLAS detector, JHEP 12 (2018) 039, arXiv: 1807.11883 [hep-ex]. 
[12] ATLAS Collaboration, Search for pair and single production of vectorlike quarks in final states with at least one $Z$ boson decaying into a pair of electrons or muons in pp collision data collected with the ATLAS detector, Phys. Rev. D 98 (2018) 112010, arXiv: 1806 . 10555 [hep-ex].

[13] ATLAS Collaboration, Search for pair production of heavy vectorlike quarks decaying into hadronic final states in pp collisions at $\sqrt{s}=13 \mathrm{TeV}$ with the ATLAS detector, Phys. Rev. D 98 (2018) 092005, arXiv: 1808.01771 [hep-ex] .

[14] ATLAS Collaboration, Search for pair production of heavy vector-like quarks decaying into high- $p_{T} W$ bosons and top quarks in the lepton-plus-jets final state in pp collisions at $\sqrt{s}=$ $13 \mathrm{TeV}$ with the ATLAS detector, JHEP 08 (2018) 048, arXiv: 1806.01762 [hep-ex] .

[15] ATLAS Collaboration, Combination of the searches for pair-produced vector-like partners of the third-generation quarks at $\sqrt{s}=13 \mathrm{TeV}$ with the ATLAS detector, Phys. Rev. Lett. 121 (2018) 211801, arXiv: 1808.02343 [hep-ex] .

[16] C. T. Hill and S. J. Parke, Top production: Sensitivity to new physics, Phys. Rev. D 49 (1994) 4454, arXiv: hep-ph/9312324 [hep-ph] .

[17] C. T. Hill, Topcolor assisted technicolor, Phys. Lett. B 345 (1995) 483, arXiv: hep-ph / 9411426 [hep-ph].

[18] A. Albert et al., Recommendations of the LHC Dark Matter Working Group: Comparing LHC searches for heavy mediators of dark matter production in visible and invisible decay channels, (2017), arXiv: 1703.05703 [hep-ex].

[19] R. M. Harris, C. T. Hill, and S. J. Parke, Cross-Section for Topcolor $Z_{t}^{\prime}$ Decaying to t $\bar{t}$, (1999), arXiv: hep-ph/9911288 [hep-ph] .

[20] B. Lillie, L. Randall, and L.-T. Wang, The Bulk RS KK-gluon at the LHC, JHEP 09 (2007) 074, arXiv: hep-ph/0701166 [hep-ph] .

[21] S. Ask, J. H. Collins, J. R. Forshaw, K. Joshi, and A. D. Pilkington, Identifying the colour of TeV-scale resonances, JHEP 01 (2012) 018, arXiv: 1108.2396 [hep-ph] .

[22] L. Randall and R. Sundrum, A Large mass hierarchy from a small extra dimension, Phys. Rev. Lett. 83 (1999) 3370, arXiv: hep-ph/9905221 [hep-ph] .

[23] K. Agashe, H. Davoudiasl, G. Perez, and A. Soni, Warped Gravitons at the LHC and Beyond, Phys. Rev. D 76 (2007) 036006, arXiv: hep-ph/ 0701186 [hep-ph] .

[24] A. L. Fitzpatrick, J. Kaplan, L. Randall, and L.-T. Wang, Searching for the Kaluza-Klein Graviton in Bulk RS Models, JHEP 09 (2007) 013, arXiv: hep-ph/ 0701150 [ hep-ph ] .

[25] ATLAS Collaboration, Search for heavy particles decaying into a top-quark pair in the fully hadronic final state in pp collisions at $\sqrt{s}=13 \mathrm{TeV}$ with the ATLAS detector, Phys. Rev. D 99 (2019) 092004, arXiv: 1902.10077 [hep-ex] .

[26] ATLAS Collaboration, Search for heavy particles decaying into top-quark pairs using leptonplus-jets events in proton-proton collisions at $\sqrt{s}=13 \mathrm{TeV}$ with the ATLAS detector, Eur. Phys. J. C 78 (2018) 565, arXiv: 1804.10823 [hep-ex ] . 
[27] ATLAS Collaboration, Boosted hadronic top identification at ATLAS for early $13 \mathrm{TeV}$ data, ATL-PHYS-PUB-2015-053, 2015, URL: https : / / cds . cern . ch/record/2116351.

[28] M. R. Buckley, T. Plehn, and M. Takeuchi, Buckets of tops, JHEP 08 (2013) 086, arXiv: 1302.6238 [hep-ph].

[29] G. Bevilacqua and M. Worek, Constraining BSM physics at the LHC: four top final states with NLO accuracy in perturbative QCD, JHEP 07 (2012) 111, arXiv: 1206.3064 [ hep-ph ] .

[30] G. Cacciapaglia, A. Deandrea, and J. Llodra-Perez, A dark matter candidate from Lorentz invariance in 6D, JHEP 03 (2010) 083, arXiv: 0907.4993 [hep-ph] .

[31] P. S. Bhupal Dev and A. Pilaftsis, Maximally Symmetric Two Higgs Doublet Model with Natural Standard Model Alignment, JHEP 12 (2014) 024, [Erratum: JHEP 11 (2015) 147], arXiv: 1408.3405 [hep-ph] .

[32] ATLAS Collaboration, Search for four-top-quark production in the single-lepton and oppositesign dilepton final states in pp collisions at $\sqrt{s}=13 \mathrm{TeV}$ with the ATLAS detector, Phys. Rev. D 99 (2019) 052009, arXiv: 1811.02305 [hep-ex] .

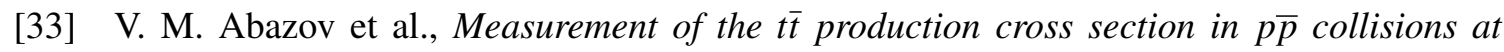
$\sqrt{s}=1.96$ TeV using secondary vertex b tagging, Phys. Rev. D 74 (11 2006) 112004, arXiv: 1902.10077 . 